\title{
The Effect of Competitive Antigen on Some Cytokine in Rabbits
}

\author{
Aseel Hashim Neama ${ }^{1}$, Ibrahim M. S. Shnawa ${ }^{2}$, Frial G. Abd ${ }^{3}$ \\ ${ }^{1}$ M.Sc., Student, ${ }^{2}$ Prof. Dr., Department of Biotechnology, College of Biotechnology, University of Qasim, Qasim \\ Babylon, Iraq, ${ }^{3}$ Prof. Dr., Department of Biology, College of Science, University of Babylon
}

\begin{abstract}
Bacterin can be divided into dead bacterin and live bacterin. Dead bacterin is a virulent strains which have a good immunogenicity .the aim of this study to know competitive between two bacterin E.coli and P.aeruginosa in different concentrations. The rabbits were used as model for this study and the rabbits were divided into six groups each groups composed 5 rabbits, After immunized programs .the blood were collected . The sera were separated and used to determine TNF- $\alpha$, IL-2 and IL-10 by enzyme linked immunosorbent assay .the results appeared .The results appeared different count of bacteria increased $(54.09,68.33) \mathrm{TNF}-\alpha$ compared with control(42.55) . the concentrations of Il-2 were increased with increased dose of $E$.coli (16.90, 20.29)also the same occurred with $P$.aeruginosa (in $P$ aeruginosa alone was 15.14 while combined with E.coli 15.56 and15.38, the results appeared the means of IL-2 concentrations were higher, Interleukin 10 were showed its mean was increased in same concentrations of bacterin but decreased in different count . The present findings seems to be novel since combined bacterins have the potential for use as an autogenous bacterins in therapy for cases of complicated multi-drug resistant infections like that of urinary tract.
\end{abstract}

Keywords: Competitive antigen, Cytokine, Rabbits, Effect

\section{Introduction}

Antigenic competition occurs as a result of competition for the limited number of immune cells with multiple capabilities already present and, on the other hand, Humoral theory believed humoral ${ }^{1}$ factors were responsible for antigenic competition and states: (Antigenic competition occurs as a result of competition for the limited amount of basic humoral factors in the immune response ${ }^{1}$. combination vaccines are composed of two or more antigens in same preperation. This method has been used in many vaccines like DTwP and MMR for over 50 years. Combination products ease the administration of vaccines and facilitate the introduction of new vaccines without requiring additional visits and injections from the health clinic.

Possible benefits of the combined vaccines include:

- Reducing the cost of storage and administration of individual vaccines

- Reducing costs for additional visits to hospitals,
- More cost-effective in vaccine delivery

- Increase immunization safety by decrease the number of needles and syringes needed

- Improving the timeliness of vaccination (some parents and health care providers object to more than two or three injectable vaccinations being given during a single visit due to a child's fear of needles and pain and safety concerns);

- Facilitating the addition of new vaccines to programmes of immunization.

The production of combination vaccines for multiple disease protection began with the combining of individual vaccines for diphtheria, tetanus, and pertussis (DTP) into one single product, this combination vaccine was first used in 1948 to vaccinate children and infants

2. It has become the fundamental of adult and pediatric immunization, over the years, has strengthened its reactogenicity profile by adding other vaccines to the mix and removing components. 
Cytokines are small secreted proteins $(<40 \mathrm{kDa})$, which are produced by nearly every cell to regulate and influence immune response ${ }^{3}$. The release of proinflammatory cytokines will lead to activation of immune cells and production as well as the release of further cytokines ${ }^{4}$

Heat-killed Brucella abortus promotes secretion of Th1-inducing cytokines such as interleukin-12 (IL12) and IFN- $\gamma$ and has been used as a carrier to induce Th1 responses to vaccines. To explore which bacterial constituents could mediate this response and how it is regulated, murine spleen cells were cultured with $B$. abortus derived DNA, lipopolysaccharide (LPS), or whole killed organismsheat-killed Brucella abortuscan also be used to stimulate Th1-like responses in mice 5. The aim of this study to determine the effect of two killed bacterial antigen on TNF-alpha, IL-10 and IL-2 concentrations in rabbits.

\section{Material and method}

\section{Preparation of Whole Heat Killed Antigen}

Escherichia coli and Pseudomonas aeruginosa isolates were obtained from Urinary tract infection patients and confirmed diagnosis of bacteria was done according to ${ }^{6}$.

The heat-killed total antigen was prepared for both bacteria when grown in a complete culture medium (mean in the heart and brain infusion broth) or a lower culture medium according to ${ }^{7}$ as follows:

1) E. coli and P. aeruginosae are grown in $100 \mathrm{ml}$ conical flasks, in which $50 \mathrm{ml}$ of the culture medium are placed and the implants are incubated in a Shaker Water

Table (1): Experimental animal groups

$\begin{array}{lll}\text { Groups } & \text { Immunization antigens } & \text { Animals number } \\ 1 & E c 1.5 * 10^{8} & 5 \\ 2 & P a 1.5 * 10^{8} & 5 \\ 3 & E c 1.5 * 10^{8}+P a 1.5 * 10^{8} & 5\end{array}$

Path vibrating bath at a temperature of $37^{\circ} \mathrm{C}$ and its tremors adjusted at a rate of 60 shake / minute and for $18 \mathrm{hr}$.

2) Discard growths are discarded at 5,000 rev / $\mathrm{min}$ for 15 mints, leaving the clearance and the precipitate taken.

3) The precipitate is suspended by a fixed volume of normal saline \& treated at $60^{\circ} \mathrm{C}$ for one hour in the water bath, the precipitate is taken and the clearance is left.

4) The precipitate was washed with functional saline and of the same original size, two washings at $5000 \mathrm{rpm}$ for $10 \mathrm{~min}$.

5) Calculate by opacity tube, the equivalent of 5 and 10 international units (by comparing the concentration of the prepared antigens with the concentration of the aforementioned tube by relying on the same degree of opacity when observed in kind).

6) It is ensured that these prepared antigens are free of bacteria that have not been killed after being treated with heat as mentioned above, where a carrier campaign taken from these antigens is grown on the feeding nest center and incubates at a temperature of $37^{\circ} \mathrm{C}$ for $18-24$ $\mathrm{hr}$. and then the growth of the colonies is observed or not. After the incubation period ends

The Immunization programs

Different concentrations \& mixtures of $E$. coli \& $P$. aeruginosaeWhole Heat Killed Antigens were used to immunize animal groups(table 1)

\section{Describe}

Injection by Only $1.5^{*} 10^{8}$ IUs of $E c$ are considered to be positive control

Injection by Only $1.5^{*} 10^{8} \mathrm{IUs}$ of $P a$ are considered to be positive control

injection with equal concentration mixture from $E c$ and $P a$ antigens $\left(1.5 * 10^{8}\right.$ IUs of each antigen) 
Cont... Table (1): Experimental animal groups

$\begin{array}{lll}4 & E c 3 * 10^{16}+P a 3 * 10^{16} & 5 \\ 5 & E c 1.5^{*} 10^{8}+P a 3 * 10^{16} & 5 \\ 6 & E c 3 * 10^{16}+P a 1.5 * 10^{8} & 5 \\ 7 & \text { Normal saline } & 5\end{array}$

\section{EC: E.coli antigen Pa: $P$. aeruginosaantigen U:Internationalunit}

\section{Blood samples}

By using disposable syringe, Five ml of blood were withdrawn from heart of the rabbits in a way of stab heart ( heart puncture), two $\mathrm{ml}$ of the samples of blood were placed in EDTA tubes as anticoagulant, while threeml of the samples of blood were placed in test tubes without anticoagulant and it was left at room temperature till being clotted, and then was centrifuged at $2500 \mathrm{rpm}$ for 5 minutes, after centrifugation the serum was separated from the blood using sterilized pasture pipette, then the serum was divided into $0.5 \mathrm{ml}$ and placed in sterilized Eppendorf tube (Freiet al.,1995) and stored at freezing till testing time.

\section{IL2,IL10,TNF Alpha}

The test was used for serum of rabbits immunized with E.coli and $P$. aeruginosae.Bacteria in in different combination to determine the concentration of IL2, IL10, TNF Alpha . This test was achieved according to the manufacturing company (Bioassay Technology Laboratory) as follows:

1- All reagents were prepared, standard solutions and samples as instructed. All reagents brought to room temperature before use. The assay was performed at room temperature.

2- The number of strips required for the assay were determined. The strips was inserted in the frames for used . injection with equal concentration mixture from $E c$ and $P a$ antigens $\left(3 * 10^{16} \mathrm{IUs}\right.$ of each antigen)

injection with antigen mixture contain $1.5^{*} 10^{8} \mathrm{IUs}$ of $E c$ and $3 * 10^{16}$ IUs of $P a$ antigens

injection with antigen mixture contain $3 * 10^{16}$ IUs of $E c$ and $1.5 * 10^{8}$ IUs of $P a$ antigens

Injection with salt solution is considered to be negative control animals

3- Fifty $\mu 1$ added from standard well.

4- Forty $\mu 1$ sample was added to sample wells and then added $10 \mu 1$ anti-IL-2 or anti-IL-10 anti-TNF Alpha (according to kit used) antibody to sample wells, then added $50 \mu \mathrm{l}$ streptavidin-HRP to sample wells and standard wells(Not blank control well). Well was mixed . The plate was covered with a sealer. Then it incubated for 60 minutes at $3^{70} \mathrm{C}$

5- The sealer was removed and washed the plate 5 times with wash buffer. wells was soaked with at least $0.35 \mathrm{ml}$ wash buffer for 30 seconds to 1 minute for each wash. For automated washing, aspirate all wells and wash 5 times with wash buffer, overfilling wells with wash buffer. Blot the plate onto paper towels or other absorbent material.

6- Fifty $\mu 1$ substrate solution A was added to each well and then added $50 \mu 1$ substrate B to each well. plate covered with a new sealer and incubated for 10 minutes at $37^{\circ} \mathrm{C}$ in the dark.

7- Fifty $\mu$ l Stop solution was added to each well, the blue color would change into yellow immediately.

8- The optical density(OD value) was determined for each well immediately using a microplate reader set to $450 \mathrm{~nm}$ within ten minutes after adding the stop solution.

\section{Statistical Analysis}

Data were processed and analyzed with oneway ANOVA using statistical program social (SPSS 23) and the results were expressed as (Mean \pm S.D). P-values below 0.05 were considered to be statistically 
significant $^{8}$.

\section{The Results and Discussion}

IL-2, IL-10and TNF-Alpha cytokines were estimated by using Enzyme Linked Immunosorbent assay (ELISA) were used for quantification of rabbit IL-2, IL-10, and TNF-Alpha the result of this test were calculated by using standard curve fit equation in ELISA system instrument . The Means of TNF-Alpha in sera of rabbit immunized with antigen(different antigen). Its mean in Groups that immunized with E.coli alone was 50while significantly decreased when combined with $P$.aeruginosa at $1.5 * 10$ was 29.24 while increased when combined in different count of bacteria was 54.09 .In Groups of rabbits that immunized with $P$.aeruginosa alone its mean was 35.49 while significantly decreased when combined with same count of E.coli 34.01 but its increased significantly when combined with different count of $E$.coli it was 68.33 . The results appeared different count of bacteria increased
(54.09, 68.33)TNF- $\alpha$ compared with control(42.55) . The cytokine tumor necrosis factor- $\alpha(\mathrm{TNF}-\alpha)$ functions as an endogenous alarm signal that coordinates gene expression and cellular activity, driving inflammatory responses to infection, injury, or irritation. In addition to stimulating host cell responses, the results appeared TNF- $\alpha$ in E.coli was higher than control while opposite with P.aeruginosa this agree with Lee and coworkers (2003) studied the in vitro and in vivo effects of TNFon two gram-negative bacteria that cause pneumonia in patients with compromised host defenses, Escherichia coli and Pseudomonas aeruginosa .E. coli responded to recombinant soluble TNF-with increased growth in vitro. This effect of TNF-on bacterial growth was dose-dependent and inhibited by blocking antibodies against TNF-. In contrast, the in vitro growth of $P$. aeruginosa was not affected by either TNF-or antiTNF- $\alpha$ antibodies. That is, recombinant TNF- $\alpha$ in vitro stimulated the growth of $E$. coli but not $P$. aeruginosa.

Table(2): The means of TNF-Alpha in serum samples of Rabbits Groups Immunized with Different Types of Antigens

\begin{tabular}{|c|c|c|c|}
\hline NO. & Groups of rabbits immunized with whole killed antigen & Mean \pm SD & P value \\
\hline 1 & E .coli $1.5 * 108$ & $50.00 \pm 9.37$ & \multirow{7}{*}{0.000} \\
\hline 2 & P. aeruginosa $1.5 * 108$ & $35.49 \pm 3.05$ & \\
\hline 3 & E.coli $(1.5 * 108)+$ P.aeruginosa $(1.5 * 108)$ & $29.24 \pm 1.12$ & \\
\hline 4 & E.coli $(3 * 1016)+$ p. aeruginosa $(3 * 1016)$ & $34.01 \pm 7.58$ & \\
\hline 5 & E.coli $(1.5 * 108)+$ p. aeruginosa $(3 * 1016)$ & $54.09 \pm 10.71$ & \\
\hline 6 & E.coli $(3 * 1016)+$ p. aeruginosa $(1.5 * 108)$ & $68.33 \pm 22.36$ & \\
\hline 7 & Control & $42.55 \pm 13.07$ & \\
\hline
\end{tabular}

The Means of IL-2in sera of rabbit immunized with antigen in table 3. E. coli alone was 10.18 while when combined with $P$.aeruginosa the concentrations of IL-2 were significantly increased .The results appeared the concentrations of Il-2 were increased with increased dose of $E$.coli $(16.90,20.29)$ also the same occurred with $P$.aeruginosa (in $P$ aeruginosa alone was15.14 while combined with E.coli 15.56 and 15.38 , the results appeared the means of IL-2 concentrations were higher significantly compared with alone bacteria . 
Table (3): The means of IL-2 in serum samples of Rabbits Groups Immunized with Different Types of Antigens

\begin{tabular}{|l|l|l|l|}
\hline NO. & Groups of rabbits immunized with whole killed antigen & Mean \pm SD & P value \\
\hline 1 & E.coli $1.5^{*} 108$ & $10.18 \pm 0.55$ & $15.14 \pm 0.78$ \\
\hline 2 & P. aeruginosa $1.5 * 108$ & $16.90 \pm 4.60$ & \multirow{2}{*}{0.001} \\
\hline 3 & E.coli1 $5^{*} 108+$ P.aeruginosa $1.5 * 108$ & $20.29 \pm 3.59$ & $15.56 \pm 1.99$ \\
\hline 4 & E.coli $3 * 1016+$ P. aeruginosa $3 * 1016$ & $15.38 \pm 1.22$ \\
\hline 5 & E.coli $1.5 * 108+$ P. aeruginosa $3 * 1016$ & $18.67 \pm 5.37$ \\
\hline 7 & E.coli $3 * 1016+$ P. aeruginosa $1.5 * 108$ & & \\
\hline
\end{tabular}

* significant at $\mathrm{p} \geq 0.05$

The Means of IL-10in sera of rabbit immunized with antigen(different antigen) ranged between (266.00-391.91) while control 293.96 .In rabbits that immunized with E.coli alone the concentrations of IL-10 was385.52while when Its concentrations(358.16) were significantly decreased combined with $P$ aeruginosa $1.5 * 10^{8}$ but increased(391.91) with $3 * 10^{16}$.In groups that immunized with $P$. aeruginosa alone its mean concentrations was 391.91 while its concentrations significantly decreased 266.00 and 281.85 (Table 4). The results were showed increased in same concentrations of bacteria but decreased in different concentrations its mean was

Table (4): The means of IL-10 in serum samples of Rabbits Groups Immunized with Different Types of Antigens

\begin{tabular}{|l|l|l|l|}
\hline NO. & Groups of rabbits immunized with whole killed antigen & Mean \pm SD & P value \\
\hline 1 & E.coli $(1.5 * 108)$ & $385.52 \pm 48.59$ & \\
\hline 2 & P. aeruginosa $(1.5 * 108)$ & $296.57 \pm 95.60$ & $358.16 \pm 20.37$ \\
\hline 3 & E.coli $(1.5 * 108)+$ P.aeruginosa $(1.5 * 108)$ & $391.91 \pm 45.87$ & 0.060 \\
\hline 4 & E.coli $(3 * 1016)+$ p. aeruginosa $(3 * 1016)$ & $266.00 \pm 37.52$ & \\
\hline 5 & E.coli $(1.5 * 108)+$ p. aeruginosa $(3 * 1016)$ & $281.85 \pm 130.54$ & $293.96 \pm 91.96$ \\
\hline 7 & E.coli $(3 * 1016)+$ p. aeruginosa $(1.5 * 108)$ & & \\
\hline
\end{tabular}

\section{*not significant at $\mathrm{p} \geq \mathbf{0 . 0 5}$}

The results showed combined bacterial effect on cytokine concentrations this disagree with9( Kashiwagi,2014 )in these study cytokine production was investigated in peripheral blood mononuclear cells (PBMCs) cultures stimulated with diphtheria and tetanus toxoids combined with acellular pertussis vaccine (DPT), Hib, and PCV7separately or 
concurrent different combinations, No significant difference was observed in cytokine levels of IL-1 $\beta$, IL-4, IL-6, IL-10, IL-12, IFN- $\gamma$, MIP-1, TNF- $\alpha$, and prostaglandin E2 (PGE2) in sera between the two groups .

Correlation between IL-10 and IL-2waspositive correlation and $p$ value was not significant at $p<0.05$ Show in figure (1), Correlation between IL-10 and TNF-Alpha was positive correlation and P value was not significant at $\mathrm{p}<0.05$ show in figure (2). Correlation between IL-2 and TNF-Alpha was positive correlation and P value was not significant at $\mathrm{p}<0.05$

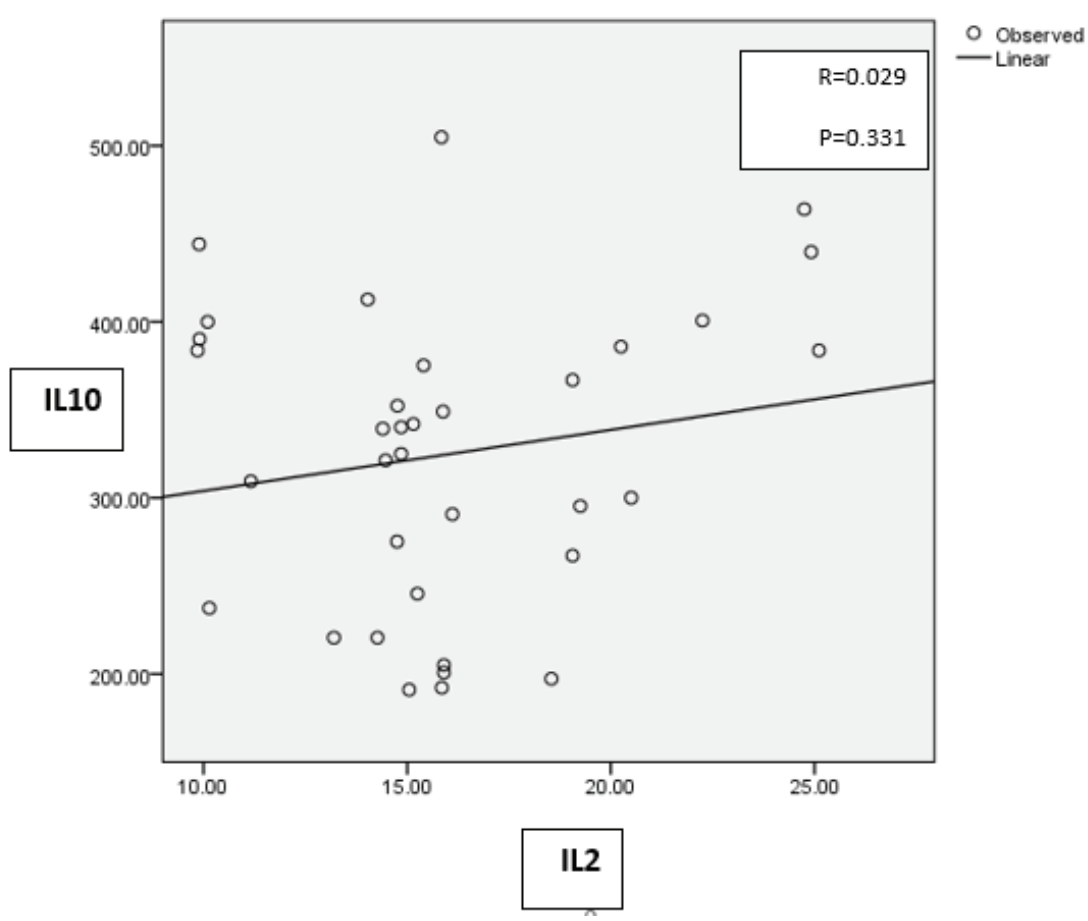

Figure(1) correlation between IL-10 and IL-2(Positive)

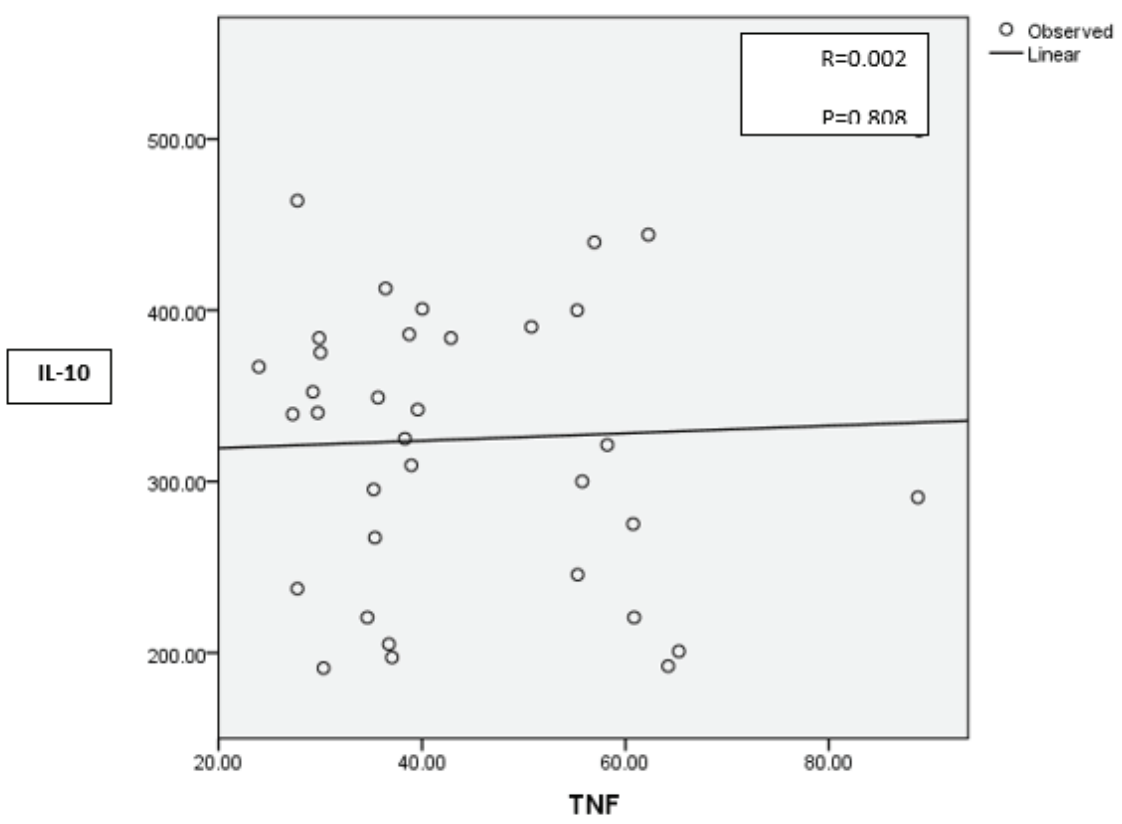

Figure (2) correlation between IL-10 and TNF-Alpha(Positive) 
Financial Disclosure: There is no financial disclosure.

Conflict of Interest: None to declare.

Ethical Clearance: All experimental protocols were approved under the College of Biotechnology and all experiments were carried out in accordance with approved guidelines.

\section{References}

1. Radovich J, Talmage D. Antigenic competition: Cellular or Humoral. Science Magazine. 1967; 158 (3800): 512-514.

2. Edwards KM, Decker MD. Vaccines. In: Plotkin SA, Orenstein WA, Offitt P, editors. Pertussis vaccine. 5th ed. USA: Saunders PA. 2008; 471528.

3. Takeuchi O, Akira S. Pattern recognition receptors and inflammation. Cell. 2010; 140: 805-820.

4. Schaper F, Rose-John S. Interleukin-6: Biology, signaling and strategies of blockade. Cytokine Growth Factor Rev. 2015; 26:475-487.

5. Hsieh CS, SE Macatonia CS. Tripp SF. Wolf A. O'Garra, and K. M.Murphy. Development of Th1 CD41T cells through IL-12produced by Listeriainduced macrophages. Science. 1993; 260:547549.

6. Forbes B A, Daniel F, Alice S. Bailey and Scott's Diagnostic Microbiology. 12th ed., Mosby Elsevier Company, USA. 2007.

7. Svanborg C, Kulhavy, R. Urinary immunoglobulins in healthy individuals and children with acute pyelonephritis. Scand. J. Immunol. 1985; 21 (4): 305-313.
8. George, A. ; Nancy, L. ; Gene, W. and Karen ,C.(2011).SPSS For Windows Step by Step. Boston, Pearson Education.IMB. International Standard Book Number: 978 0-415-88229-3.

9. Feri, J. ; Heuck, C. ; Riesen, W . and Laug, I.T.(1995).Production of Basic Diagnosis Laboratory Reagents. WHO regional publication. Eastern Mealiterranean Series.

10. Huang L.; Krieg AM.; Eller N.; Scott DE.(1999) Induction and regulation of Th1-inducing cytokines by bacterial DNA, lipopolysaccharide, and heatinactivated bacteria. Infection and Immunity, 67(12). 6257-6263.

11. Kashiwagi Y, Miyata A. Production of inflammatory cytokines in response to diphtheriapertussis-tetanus (DPT), haemophilus influenzae type b (Hib), and 7-valent pneumococcal (PCV7) vaccines .Human Vaccines \& Immunotherapeutics. 2014; 10:3, 677.

12. Lee J-H, Del Sorbo L, Khine AA, de Azavedo J, Low DE, Bell D, UhligS, Slutsky AS, Zhang H. Modulation of bacterial growth by tumornecrosis factor-in vitroandin vivo. Am J Respir Crit Care Med. 2003; 168: 1462-1470.

13. MacFadden J. Biochemical tests for Identification of Medical Bacteria. 3rd ed. The Williams and Wilkins Co., USA. 2000: 689- 691

14. World Health Organization (WHO).( 2020) "Module 2: type of vaccine and adverse reaction Immunization in Practice, WHO/IVB/04.06, Geneva: WHO, 2020. 